\title{
Application of an Intra-operative Load Measuring System for Knee Replacement Surgery
}

\author{
T.V. Skrinskas ${ }^{1,2}$, D.G. Viskontas ${ }^{1,3}$, L. Ferreira ${ }^{1}$, D.G. Chess ${ }^{1,3}$, and J.A. Johnson ${ }^{1,2,3,4}$ \\ ${ }^{1}$ Hand and Upper Limb Centre, Bioengineering Laboratory, \\ St. Joseph's Health Care London, \\ 268 Grosvenor St., London, ON, Canada, N6A 4L6 \\ ${ }^{2}$ Department of Biomedical Engineering, \\ ${ }^{3}$ Department of Surgery, \\ ${ }^{4}$ Department, Mechanical and Materials Engineering \\ University of Western Ontario, London, ON, Canada \\ jajohnson@uwo.ca
}

\begin{abstract}
Joint gap balancing during total knee arthroplasty has been an empirical undertaking to date. Ligament tensioning devices exist to aid soft tissue management, however quantifying tension remains problematic. The purpose of this study was to validate modifications made to a current balancing system, allowing for quantification of joint load using a computer-assisted technique. An additional goal was to determine if the tool would improve joint gap management and consequently load balance in an in-vitro model. The load sensing capability of the tool was validated under known loading conditions. Intraoperative use of the tool relative to current techniques was evaluated in a preliminary in-vitro study using cadaveric knees. There was a trend towards improved joint gap management when using the tensioning device, but this did not translate into a more balanced joint in our knee simulator.
\end{abstract}

\section{Introduction}

The success of total knee arthroplasty (TKA) is, in all likelihood, dependent on the joint alignment and balance achieved at the time of surgery. To date, perfect soft tissue balance has been difficult to achieve [1]. The goal is to create equal tension in the medial and lateral ligaments. The difficulty is not balancing the knee at any one discrete flexion angle, but in creating equal tension between the sides throughout the entire range of motion. This is challenging since gap changes related to release are more pronounced in flexion than in extension $[2,3,4]$. Often the surgeon is forced to compromise.

While balancing is currently done qualitatively in the operating theatre, instrumentation has been developed to quantify imbalance. One system is the Stryker Xcelerate knee balancer in Figure 1.(Stryker-Howmedica-Osteonics, NJ). This tool functions on the principle of distracting the joint and tensioning the ligaments. The two main features are its ability to distract the joint via a ratcheting system and to measure ligament imbalance as the angulation of a pivoting plate that contacts the femoral condyles $[5,6]$. 


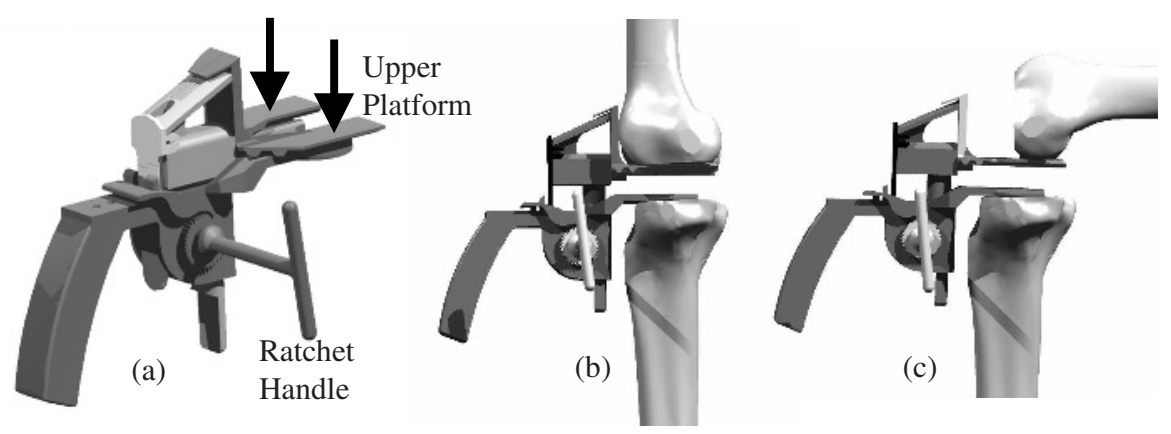

Fig. 1. A currently available balancer tool $(a)$. The tool distracts and tensions the ligaments in the extended $(b)$ and flexed $(c)$ joint. Loads in either compartment, an indicated by the vectors in $(a)$, are balanced through appropriate ligament releases.

The tensioning device helped guide balance in a study to determine if proprioception is affected by soft-tissue deformity in 38 patients [7]. More recently, the device achieved results comparable to traditional methods in a clinical study [8]. There remain limitations to the system including the application of an "appropriate" tension to the ligaments by the surgeon. The joint distraction space and angulation have been acquired electronically in the past [5], although these are not standard features on the commercially available unit. Moreover, there is a lack of biomechanical studies of surgical outcomes using the tool versus traditional methods with a specific interest in joint load.

In light of the foregoing, the purpose of this study was to validate the addition of a computer assisted load-sensing system to the Stryker Xcelerate tensioning device. An additional goal was to determine if the tool could improve joint gap management, and consequently load balance, in an in-vitro model compared to traditional balancing and resection techniques.

\section{Modifications}

An assembly consisting of two beams, two hinge joints, a mounting bracket and four load cells was added to the upper platform of the tensioning device. On either side of the upper platform, each beam housed two load cells and was joined via a hinge to the bracket. These additions measured the compressive load on either side of the upper platform (i.e. in the medial and lateral compartments) (Fig. 2).

The load cells are strain gauge based compression cells (Model 13, 50 lbs capacity, Sensotec, Columbus, OH). Signal conditioning (SCXI) and data acquisition hardware (DAQ) (National Instruments, Austin, TX) were used to process the load cell outputs. The cells were wired into two SCXI 1321 terminal blocks that were in turn connected to SCXI 1121 modules. These provided signal conditioning and an excitation voltage of $3.33 \mathrm{~V}$ to the cells. The modules were grouped into an SCXI 1001 chassis. A PCIMIO-16E DAQ card monitored the signals from the chassis. A custom written LabVIEW program (National Instruments, Austin, TX) converted the final processed voltage signal to the load measured by each cell (Fig. 3). 


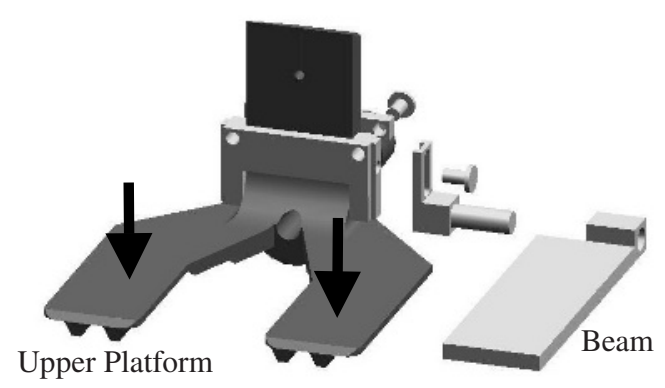

(a)

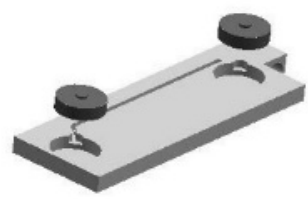

(b)

Fig. 2. Modifications made to the top platform of the tensioning device: $(a)$ exploded view of the beam assembly on one side of the upper platform, $(b)$ underside of the beam that houses the two miniature load cells (wiring not shown).

Using equilibrium and deflection analysis of the beam, equations were developed to predict the resultant output load on each condyle. A calibration was performed to relate this output to a known load. The calibration apparatus consisted of a pneumatic actuator and load cell mounted to a frame in a position to apply a pure known compressive load.

A series of incremental loads were applied in $5 \mathrm{~mm}$ increments between 15 and 55 $\mathrm{mm}$ from the hinge. The positions along the beam were measured with digital calipers. Two relationships for position and load were cross-referenced to obtain the final value of an unknown applied force. The LabVIEW program performed all the necessary calculations and provided real-time feedback of the measured values (Fig. 3).

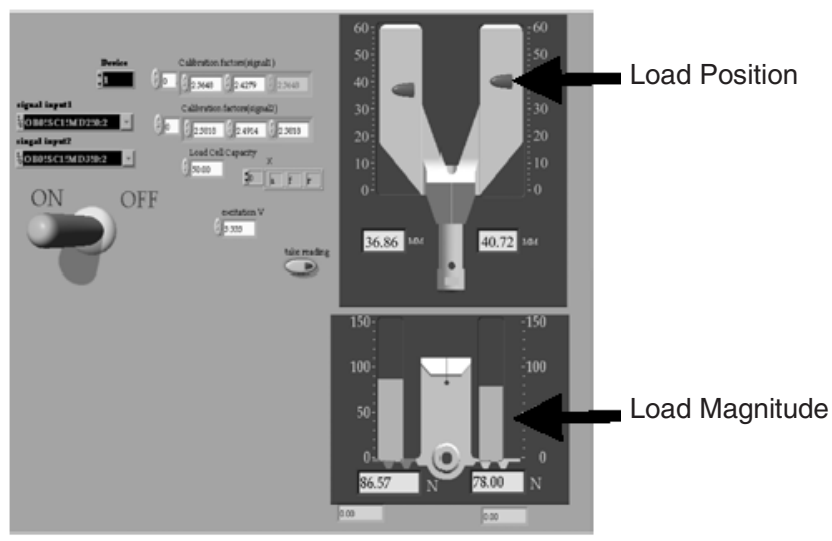

Fig. 3. Graphical User Interface (GUI) of the program monitoring the joint loads (bottom right) on either side of the platform and their position (top right). 


\section{Methods}

\subsection{Tensioning Device}

The experimental set-up of the calibration was also used to study the repeatability, hysteresis and accuracy of the load sensing beams. For all tests, the magnitude and position of the applied force were monitored while the measured load and position were recorded.

To test repeatability, a series of incremental loads between 27 and $110 \mathrm{~N}$ were applied at positions of 20,30, 40 and $50 \mathrm{~mm}$ from the hinge. This procedure was repeated five times. Hysteresis of the system was tested at 2 distances along the beam, 30 and $50 \mathrm{~mm}$. Incremental loads were applied from $27 \mathrm{~N}$ to $110 \mathrm{~N}$ and decremented in the same fashion. Accuracy was determined for both sides of the platform. Ten random loads were applied each at a distinct and randomly chosen position along the beam. A linear regression analysis was performed to evaluate repeatability and accuracy.

\subsection{In vitro Testing}

Four pairs of fresh-frozen cadaveric knees were tested (mean age $69 \pm 19$ years). The specimens were amputated $20 \mathrm{~cm}$ proximal to the distal femoral condyles and $25 \mathrm{~cm}$ distal to the tibial plateau. Each femur was clamped into our custom knee simulator [9]. Knee replacement surgery (Scorpio Knee System, Stryker-HowmedicaOsteonics, NJ) was performed on the specimens using a traditional surgical approach on the control side and an experimental protocol on the contralateral knee. Soft tissue balancing in the controls was qualitative and bone cuts were performed using commercial alignment jigs. The femur was prepared first with the distal femoral resection set at $6^{\circ}$ of valgus and femoral rotation determined relative to the epicondylar axis. The tibial plateau was resected perpendicular to the shaft axis. Ligament balancing was then performed accordingly by visual inspection. In the experimental knees, the surgical protocol was customized to use the modified tensioning device and the commercial jigs. The distal femoral and tibial resections were performed first in the standard manner. Ligament balancing was done in the extended joint using the tensioning device at a load between 170 and $200 \mathrm{~N}$. The knee was brought into flexion and distracted to approximately the same force. The posterior condyles were resected along a plane parallel to the tibial plateau using custom designed jigs to work in conjunction with the tensioning device. Joint loads between extension and flexion were then equalized with appropriate residual resections. Before cementing the components, the load and gap alignment at four gap distractions were measured in the flexed joint using the tensioning device. Zero degrees of angulation was considered a perfect gap alignment.

Following surgery, the quadriceps and hamstring muscles were loaded to $200 \mathrm{~N}$ and $80 \mathrm{~N}$ respectively [10]. The loading protocol was performed at 5 discrete flexion angles $\left(0^{\circ}, 30^{\circ}, 45^{\circ}, 60^{\circ}, 90^{\circ}\right)$. The knee was otherwise unconstrained and free to move in varus/valgus angulation and internal/external rotation. The compressive forces in the medial and lateral compartments of the knee were recorded by a tibial load transducer initially developed in our laboratory [11] and further improved for this study. 
Surgical outcome was evaluated by inspecting the imbalance in the joint. Imbalance was defined as the difference in loads between the medial and lateral compartments over the net load in the joint. Data was anaylzed using a two-way repeated measures $\operatorname{ANOVA}(\alpha=0.05)$.

\section{Results}

During repeatability trials of the tensioning device, the measured load values correlated well with the applied load $\left(\mathrm{R}^{2}=0.997, \mathrm{P}<0.001\right.$, slope $\left.\mathrm{m}=0.99\right)$ with a maximum standard deviation of $5.7 \mathrm{~N}$. Both load-sensing beams on either side of the tensioning device performed equally well in the accuracy testing, therefore results will be given for only one side in Figure $4\left(\mathrm{R}^{2}=0.987, \mathrm{P}<0.001\right.$, slope $\left.\mathrm{m}=0.90\right)$. The average load error was $2.6 \mathrm{~N}$. Hysteresis effects were not significant with a maximum difference between the incremental and decremental loading curves of $8 \mathrm{~N}$.

There was no difference between the surgical protocols when comparing their ability to ensure equal tensioning load in the extended and flexed joint gaps $(\mathrm{p}=0.3)$. There was also no difference in tensioning load between the extended and flexed positions regardless of surgical protocol $(p=0.5)$. The groups had the same flexion gap alignment $(\mathrm{p}=0.3)$ and neither group had an alignment significantly different than $0^{\circ}$ $(\mathrm{p}=0.3$, controls; $\mathrm{p}=0.7$, experimental). However, there was a tendency towards a more balanced flexion gap when using the tensioning device (Fig. 5).

The experimental protocol did not improve joint balance relative to traditional methods $(\mathrm{p}=0.9)$ when observing compartmental force in the joint under simulated muscle loading (Fig. 6). There was a significant increase in imbalance with increasing flexion angle $(\mathrm{p}=0.046)$.

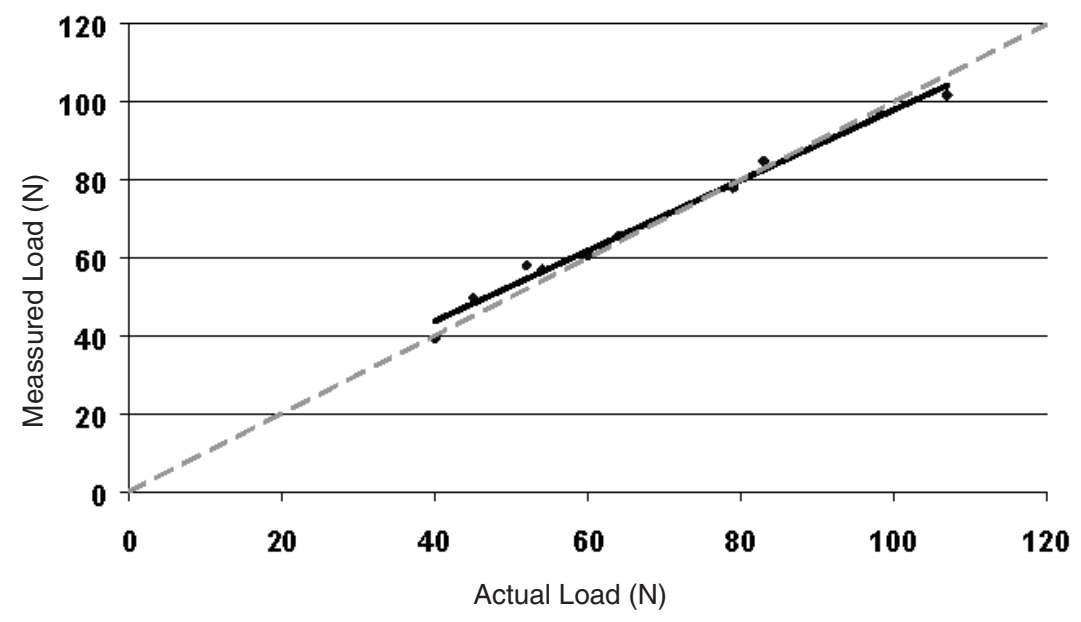

Fig. 4. A plot of actual load (horizontal axis) versus measured load (vertical axis) to determine the accuracy of the load measuring system $\left(\mathrm{R}^{2}=0.987, \mathrm{P}<0.001\right.$, slope $\left.\mathrm{m}=0.90\right)$ 


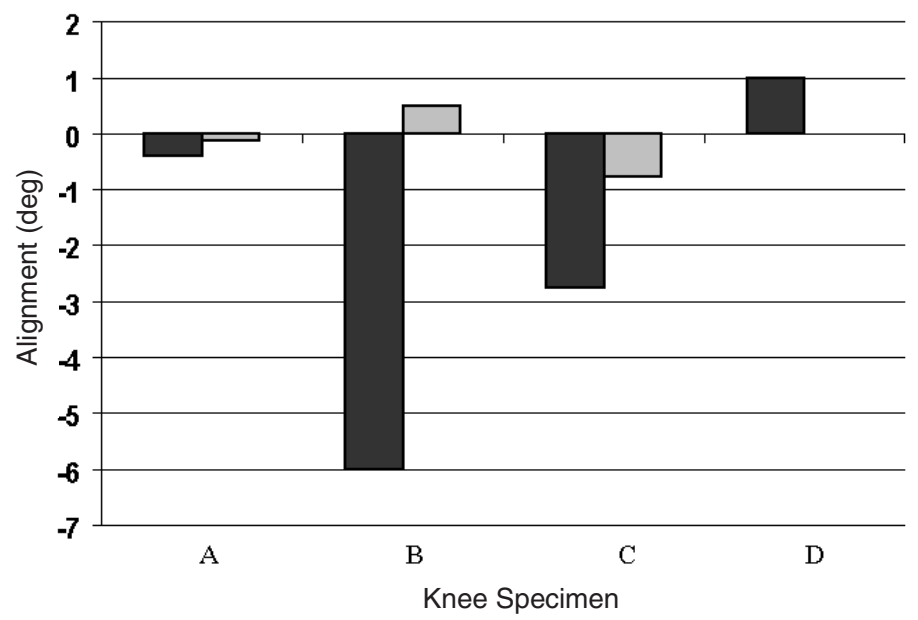

Fig. 5. This graph shows the average flexion gap alignment (vertical axis) over the four distractions for the four knee specimens (horizontal axis). The experimental side (gray) shows a trend towards a more balanced gap relative to the controls (black). Specimen D had a perfect alignment in the experimental side

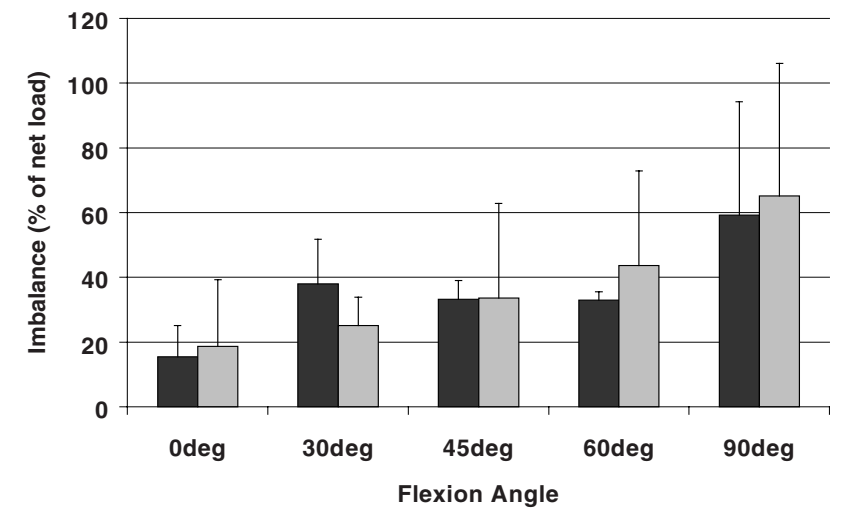

Fig. 6. This graph summarizes the mean and standard deviations of the imbalance in the knee joint ( $y$-axis) with increasing flexion angle $(x$-axis) for the four pairs of knees. There is no difference between the controls (black) and the experimental knees (gray) $(\mathrm{P}=0.9)$, however imbalance did increase with flexion angle $(\mathrm{P}=0.046)$

\section{Discussion}

In this study, load-sensing modifications to a currently available knee balancer were validated. The results strongly support the accuracy and repeatability of the system. Our system is an improvement relative to standard tools, as they are unable to quantify the load that is applied to the joint. This limitation of the Stryker tool has been 
noted in the past in Winemaker's clinical study [8]. Currently, the commercially available unit can be instrumented with a torque meter on the ratchet handle though there is no literature to date reporting on the use of the load feedback in surgical trials.

Our aim was also to determine if the tensioning device improved joint gap preparation and consequently load balance compared to traditional methods in an in-vitro model. A properly balanced joint gap has two characteristics. First, the joint space must be square in flexion and extension. Second, the gaps must be of equal distracted size under the same tensioning load. The trends in the data support the use of the tensioning device in accomplishing the first criteria of proper gap preparation. Figure 5 depicts a clear tendency towards alignment balance for the experimental group compared to the controls. However, the results indicate that the surgical protocol may not affect tensioning load balance between the extended and flexed joint. This is the first study to evaluate this aspect of gap management. Current techniques for determining the size of the joint gap involve tracking the amount of bone that is resected and visually inspecting the joint space. The load data indicates that this method is comparable to the management directed by the tool.

When considering the final balance of the joint after implant cementation, the two groups are not significantly different (Fig. 6). The increased imbalance with flexion angle can be attributed to the rotational alignment of the femoral component. This highlights the difficulty in achieving a balanced joint throughout a full range of motion and fuels the debate on proper gap preparation, particularly rotational alignment of the femoral component. Imposing a femoral rotation that creates a square gap when ligaments are stressed is a technique that has been recently addressed [8], [12], [13]. The interdependence of rotational alignment and soft tissue balance in flexion has long been acknowledged, though only recently has ligament tension been suggested as the definitive parameter in deciding femoral rotation.

In summary, this study has shown that the use of computer-assisted balancing in knee replacement surgery appears efficacious from the viewpoint of improving joint gap preparation and providing the surgical team with real-time feedback of joint alignment and loading. Further work will include incorporating ligament balancing protocols with this computer-assisted instrumentation.

\section{References}

1. Griffin FM, Insall JN, Scuderi GR: Accuracy of soft tissue balancing in total knee arthroplasty. J Arthroplasty 15: 970-973, 2000

2. Krackow KA, Mihalko WM: The effect of medial release on flexion and extension gaps in cadaveric knees: implications for soft-tissue balancing in total knee arthroplasty. Am J Knee Surg 12: 222-228, 1999

3. Krackow KA, Mihalko WM: Flexion-extension joint gap changes after lateral structure release for valgus deformity correction in total knee arthroplasty: a cadaveric study. J Arthroplasty 14: 994-1004, 1999

4. Mihalko WM, Miller C, Krackow KA: Total knee arthroplasty ligament balancing and gap kinematics with posterior cruciate ligament retention and sacrifice. Am J Orthop 29: 610-616, 2000

5. Attfield SF, Warren-Forward M, Wilton T, Sambatakakis A: Measurement of soft tissue imbalance in total knee arthroplasty using electronic instrumentation. Med Eng Phys 16: 501-505, 1994 
6. Sambatakakis A, Attfield SF, Newton G: Quantification of soft-tissue imbalance in condylar knee arthroplasty. J Biomed Eng 15: 339-343, 1993

7. Attfield SF, Wilton TJ, Pratt DJ, Sambatakakis A: Soft-tissue balance and recovery of proprioception after total knee replacement. J Bone Joint Surg Br 78: 540-545, 1996

8. Winemaker MJ: Perfect balance in total knee arthroplasty: the elusive compromise. J Arthroplasty 17: 2-10, 2002

9. El-Hawary R, Roth SE, Harwood C, Johnson JA, King GJ, Chess DG: The Effect of the Posterior Cruciate Ligament on Total Knee Arthroplasty Load Balance. 49th Annual Meeting, Orthopaedic Research Society 2003

10. Li G, Rudy TW, Sakane M, Kanamori A, Ma CB, Woo SL: The importance of quadriceps and hamstring muscle loading on knee kinematics and in-situ forces in the ACL. J Biomech 32: 395-400, 1999

11. Roth SE, Chess DG, Dunning C, Johnson JA, King GJ: Design of a Transducer to Measure Intra-Operative Loading During Total Knee Replacement. 35th Annual Meeting, Canadian Orthopedic Research Society 2001

12. Kunz M, Strauss M, Langlotz F, Deuretzbacher G, Ruther W, Nolte L-P: A non-CT based total knee arthroplasty system featuring complete soft-tissue balancing. 4th Annual MICCAI Meeting 2001

13. Stiehl JB, Cherveny PM: Femoral rotational alignment using the tibial shaft axis in total knee arthroplasty. Clin Orthop 47-55, 1996 